\title{
The Design of Cavity Resonators and Microwave Filters Applying Shape Deformation Techniques
}

This paper was downloaded from TechRxiv (https://www.techrxiv.org).

\section{LICENSE}

CC BY-NC-SA 4.0

SUBMISSION DATE / POSTED DATE

03-02-2022 / 07-02-2022

\section{CITATION}

Baranowski, Michal; Balewski, Łukasz; Lamecki, Adam; Mrozowski, Michal; Galdeano, Jaione (2022): The Design of Cavity Resonators and Microwave Filters Applying Shape Deformation Techniques. TechRxiv. Preprint. https://doi.org/10.36227/techrxiv.19114457.v1

DOI

10.36227/techrxiv.19114457.v1 


\title{
The Design of Cavity Resonators and Microwave Filters Applying Shape Deformation Techniques
}

\author{
Michal Baranowski, Graduate Student Member, IEEE, Łukasz Balewski, Adam Lamecki, Senior Member, IEEE, \\ Michal Mrozowski, Fellow, IEEE and Jaione Galdeano
}

\begin{abstract}
This paper introduces shape deformation as a new approach to the computer-aided design (CAD) of high-frequency components. We show that geometry deformation opens up new design possibilities and offers additional degrees of freedom in the 3D modeling of microwave structures. Such design flexibility is highly desirable if the full potential of Additive Manufacturing (AM) is to be exploited in the fabrication of RF \& microwave devices. The use of deformation techniques in the design of highfrequency components allows the attainment of improved electrical parameters, such as high-quality factors for cavity resonators and wide higher-order mode separation. In this work, shape deformation with radial basis functions (RBFs) is integrated with an electromagnetic field simulator based on the 3D finiteelement method (FEM), allowing the semiautomated optimization of microwave components, such as cavity resonators and filters. The proposed strategy is used for the design of high Q-factor cavity resonators and of cavity bandpass filters with improved spurious mode separation. Designs of third-order and sixth-order cavity filters with complex geometry are experimentally verified using 3D-printed prototypes with selective laser melting (SLM) technology.
\end{abstract}

Index Terms - cavity resonator, CAD, design optimization, shape deformation, radial basis functions, additive manufacturing, microwave filter.

\section{INTRODUCTION}

The use of additive manufacturing technology (also known as $3 \mathrm{D}$ printing) to fabricate high-frequency components has been increasing in popularity in recent years [1]-[4]. AM allows for the production of complex 3D structures of almost arbitrary shapes, with user-defined surface curvatures. This flexibility in model complexity is a great advantage over established technologies, as the limitations of conventional techniques such as CNC milling no longer apply. In many cases, AM permits the production of a model that would not be achievable using other manufacturing methods. In this context, AM is a key enabling technology that opens up new

M. Baranowski, A. Lamecki, and M. Mrozowski are with the Faculty of Electronics, Telecommunications, and Informatics, Gdańsk University of Technology, 80-233 Gdańsk, Poland (e-mail: m.baranowski@ieee.org; adam.lamecki@ieee.org; m.mrozowski@ieee.org).

Ł. Balewski and A. Lamecki are with EM Invent Sp. $\mathrm{z}$ o.o. (limited company), Trzy Lipy 3, 80-172 Gdańsk, Poland (e-mail: lukasz.balewski@eminvent.com).

J. Galdeano is with the European Space Research and Technology Center, European Space Agency, 2201 AZ Noordwijk, The Netherlands (e-mail: jaione.galdeano@esa.int).

This work was supported by the National Science Centre, Poland under agreement 2020/39/O/ST7/02897 and the European Space Agency under contract 4000128097/19/NL/CBi.

This paper is an expanded version of a paper presented at the IEEE MTT-S International Microwave Filter Workshop, Perugia, Italy 17-19 Nov. 2021. possibilities to RF \& microwave engineers. However, in order to take advantage of this design flexibility, the limitations of the CAD tools currently used in the industry need to be overcome. These limitations stem from the fact that virtually all CAD software used for high-frequency electronic design relies on the constructive solid geometry (CSG) approach. CSG is the prevailing paradigm for the 3D modeling of electromagnetic structures. It is based on using a set of simple, predefined geometrical primitives-such as boxes, cylinders, and cones-and applying to them Boolean operations, such as union, subtraction, and intersection, or shape modifications with edge filleting or chamfering. Alternatively, arbitrarily shaped surfaces based on parametric, equation-based objects can be used, but this is cumbersome and difficult to use. The design of more complex geometrical models-such as those with unconventional twisted or bent shapes-is very challenging in this approach. For decades, the CSG approach has been regarded as satisfactory, as design was limited by the available manufacturing technologies, like $\mathrm{CNC}$ milling. This has now changed with the widespread availability of repeatable and accurate $3 \mathrm{D}$ printing.

To date, many novel microwave components have been developed using AM. However, when these designs are examined, it becomes clear that designers are still restricted by the limited capabilities of 3D EM tools based on CSG. A literature review shows that all designs using 3D printing technology for RF \& microwave components have followed the CSG approach [5]-[9]. The development of new CAD tools is thus essential if the full potential of AM in microwave technologies is to be unleashed. The present work can be considered a step towards achieving this goal; to enable the design of microwave components with truly arbitrary shapes, our proposed solution is to empower CSG-based 3D solid modeling tools with a free-form shape deformation technique.

Geometry deformation can be treated as an additional step in the design process. It can be performed on objects created in CAD tools with CSG. The roots of shape deformation techniques lie in computer graphics applications, where they are often used for object manipulation. One of the first deformation techniques was free-form deformation (FFD), proposed by Sederberg and Parry in 1986 [10]. Several other methods were developed later, including FFD based on nonuniform rational B-splines (NURBS) [11], [12]. Recently, shape deformation has been introduced into computational simulations of fluid dynamics [13]-[15] and low-frequency electromagnetics [16]. Some attempts to shape optimization using, e.g., level-set and topology gradient methods and Bézier curve parametrization 

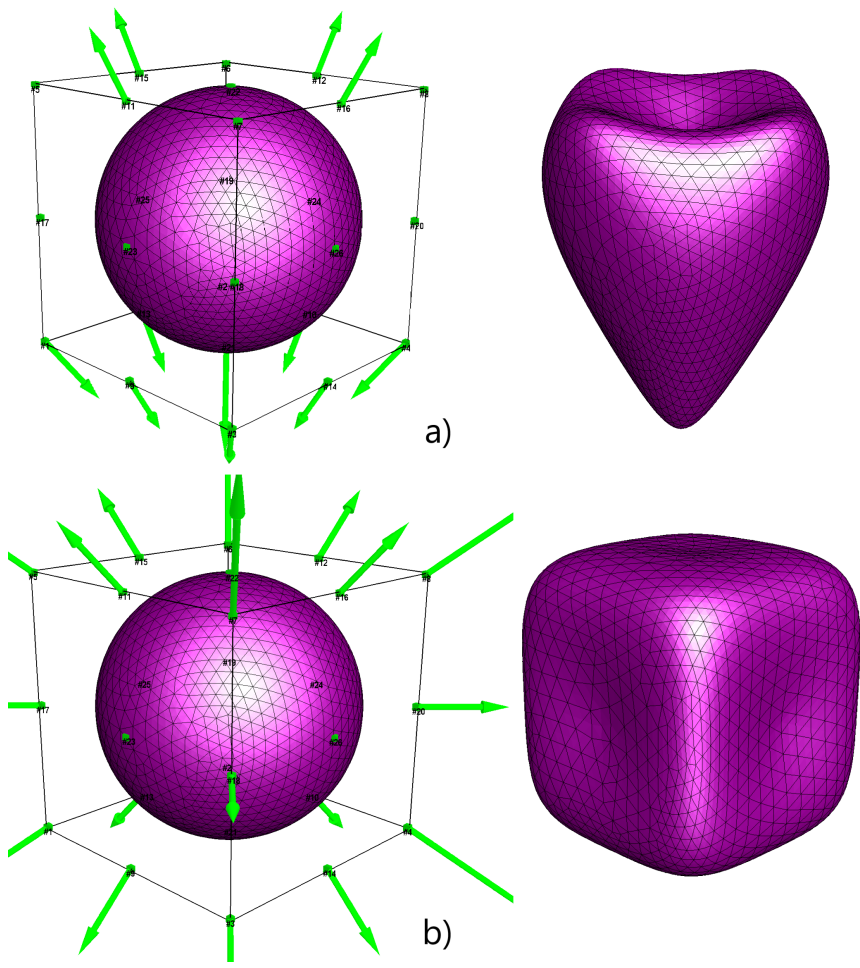

Fig. 1. Two examples of different shape deformations performed on the same spherical object. Left: the initial sphere; right: the result of the deforming operation. Control points and their displacements are represented by green points and arrows.

have been reported for the design of microwave components, [17]-[20] but as far as we know, no attempts have yet been made to use shape deformation techniques to model complex 3D microwave structures.

The basic principle of FFD is to define a set of points around the object that will control the deforming operation. The deformation is then begun by shifting some of these control points to certain new coordinates, and a vector displacement field is constructed using these displacements. The original structure is then mapped according to this smooth displacement function. The deforming operation can be performed on 3D objects represented by a surface or volume mesh. In this work, we consider a tetrahedral mesh used in 3D FEM. Two examples of different geometry deformation operations, shown in Fig. 1 a and Fig. 1p, illustrate how the shape deformation method can be applied to $3 \mathrm{D}$ objects. It can be seen that, by starting from a basic shape and simply moving the control points in different directions, various complex geometries can be obtained. The continuity of the object's surface (topology) and its smoothness are also preserved.

In this paper, shape deformation is integrated into the design process of high-frequency electronics. Mesh deformations are coupled with the 3D FEM electromagnetic field simulator InventSim [21], allowing for the semiautomated design of various microwave components. The deformation operation is executed as an additional step in the EM simulation process, directly after the mesh of the analyzed structure is generated. The aim of this work is to show that this approach can remove current design limitations, and thus improve control over the electrical parameters of structures, such as unloaded quality factor and higher-order mode separation of cavity resonators and filters. The improvement of these parameters is highly desirable, e.g., in microwave filters for space applications [1].

In this paper, the idea presented in [22] has been further developed and extended. Shape deformation with radial basis functions is described in detail in Section II, along with several illustrative examples. A general framework for design optimization using these deformation techniques is presented in Section III. An investigation of cavity resonators exploiting geometry deformations for the the Q-factor and spuriousfree performance is given in Section IV. An extension of this concept is provided in Section $\mathrm{V}$, featuring the design of deformed waveguide cavity filters. Prototypes of thirdorder and sixth-order bandpass filters are fabricated using AM, and the measurement results are compared with simulations. Finally, the concluding remarks are given in Section VI.

\section{SHAPE DEFORMATION WITH RBFS}

Shape deformation is implemented here using interpolation with radial basis functions (RBF), as reported in [13], [23][25]. In general, a RBF is a real-valued function $\varphi$ whose value is only determined by the distance from the origin, so that $\varphi(\boldsymbol{x})=\hat{\varphi}(\|\boldsymbol{x}\|)$. The principle of the reshaping operation is represented in general by a mapping function $\mathcal{D}$ :

$$
\begin{aligned}
\mathcal{D}(\boldsymbol{x}): \mathbb{R}^{3} & \rightarrow \mathbb{R}^{3} \\
\boldsymbol{x} & \mapsto \mathcal{D}(\boldsymbol{x})=\boldsymbol{x}+\boldsymbol{d}(\boldsymbol{x})
\end{aligned}
$$

In this notation, $\boldsymbol{d}(\boldsymbol{x})$ can be interpreted as the deformation field applied to the 3D model. In the case of RBF-based deformation, this takes the following form:

$$
\boldsymbol{d}(\boldsymbol{x})=\sum_{i=1}^{N} \gamma_{\boldsymbol{i}} \varphi\left(\left\|\boldsymbol{x}-\boldsymbol{x}_{\boldsymbol{i}}\right\|\right)+p(\boldsymbol{x})
$$

where $N$ is the number of control points defining the deformation, $\gamma_{i}$ are the weights corresponding to the radial basis functions $\varphi$ defined at the positions of the control points $\boldsymbol{x}_{i}$ (in 3D $\boldsymbol{x}_{\boldsymbol{i}} \in \mathbb{R}^{3}, \boldsymbol{x}_{\boldsymbol{i}}=\left[x_{i}, y_{i}, z_{i}\right]$ ), and $p(\boldsymbol{x})$ is a low-order polynomial (optional).

To perform the deformation, the mapping operation $\mathcal{D}$ needs to be specified. The first step is to define a set of $N$ control points and their displacements, which indicate the direction and value of the offset. Next, a certain type of RBF with its parameters (e.g., radius, exponent) is selected: the choice of basis function affects the manner in which the mesh points are interpolated to match the new positions of the control points. There is a variety of RBFs commonly used for multivariate function interpolation, such as:

- Gaussian spline: $\varphi(\|\boldsymbol{x}\|)=\exp \left(-\left(\frac{\|\boldsymbol{x}\|}{r}\right)^{2}\right)$,

- multi-quadratic biharmonic spline:

$$
\varphi(\|\boldsymbol{x}\|)=\sqrt{\|\boldsymbol{x}\|^{2}+r^{2}},
$$

- polyharmonic spline:

$$
\varphi(\|\boldsymbol{x}\|)=\left\{\begin{array}{ll}
\left(\frac{\|\boldsymbol{x}\|}{r}\right)^{k} & \text { if } k=1,3,5, \ldots \\
\left(\frac{\|\boldsymbol{x}\|}{r}\right)^{k} \ln \left(\frac{\|\boldsymbol{x}\|}{r}\right) & \text { if } k=2,4,6, \ldots
\end{array},\right.
$$


where $r$ and $k$ are the radius and power of the RBF, respectively. A linear equation system is then solved to find the unknown weights $\gamma_{i}$ given the coordinates of the original and displaced control points [24]. The matrix representation of this equation system is as follows:

$$
\Phi \gamma=B
$$

where $\gamma$ is the weight matrix of dimension $N \times 3$, corresponding to $N$ control points defined in a 3D coordinate system. $\Phi$ is an $N \times N$ matrix containing RBF values computed for the Euclidean distances between the corresponding control points, $\boldsymbol{\Phi}_{\boldsymbol{i}, \boldsymbol{j}}=\varphi\left(\left\|\boldsymbol{x}_{\boldsymbol{i}}-\boldsymbol{x}_{\boldsymbol{j}}\right\|\right)$, and $\boldsymbol{B}$ is an $N \times 3$ right-hand side matrix with the new (displaced) coordinates of the control points. The system is dense, symmetric, positive definite, and its conditioning depends on selected RBF and control points distribution. Once the weights are computed, the deformation field $\boldsymbol{d}(\boldsymbol{x})$ is constructed and can be applied to smoothly move all the mesh points of a 3D object. It can be seen that the mapping function can be easily defined with a limited number of control points, and then applied to create complex $3 \mathrm{D}$ objects. The computational cost of the deformation is also not significant, as the size of the linear problem to be solved is usually small. To illustrate this framework, we will take the simple example of the sphere presented in Fig. 1 . In order to alter the geometry of this structure, a number of control points are selected around it. In this example, the control points are defined on a box enclosing the sphere. There are 26 control points in total: eight points at the vertices, six points at the center of each face, and twelve points at the midpoints of each edge. The displacement vectors are then defined for some of these points. Depending on the direction and number of control point offsets, the original shape is deformed to produce a different final geometry. It should be noted that the shape deformation scheme does not limit the number of control points. More complex deformation schemes can easily be employed by adding more control points with movement in arbitrary directions. Additionally, the definition of object deformation through displacement vectors of predefined control points is intuitive, and its result is easy to predict, as the deformation follows the displacements.

The technique can also be applied to bend or twist complex objects, as shown for the example of a waveguide cavity filter in Fig. 2. We can also define a series of deforming operations which can be executed one after another, such as squeezing, twisting, and bending, as shown in Fig. 2 e.

\section{General Framework FOR DESIGN Optimization WITH SHAPE DEFORMATION}

Shape deformation can be easily integrated with design optimization, enriching the standard design parametrization with a new set of design variables that define the deformation operation-namely the positions and movements of selected control points. The proposed design procedure is shown in the block diagram in Fig. 3. The optimization loop involves managing two sets of design variables: geometrical parameters altering the basic shape of the primal structure that is constructed using CSG, and deformation parameters controlling

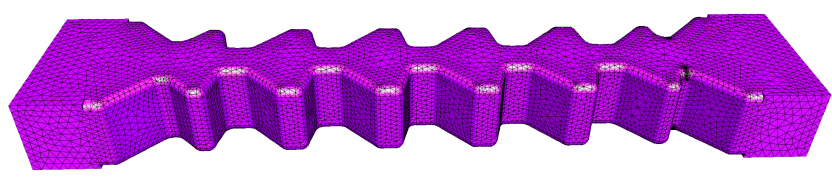

a)

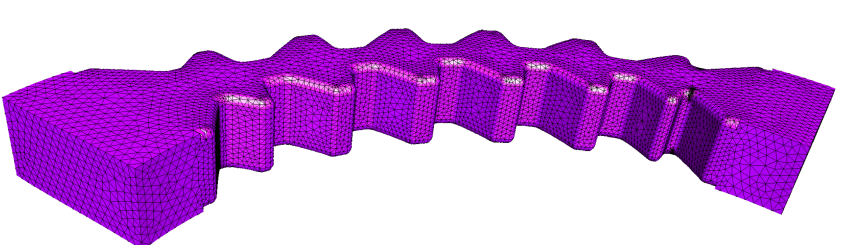

b)
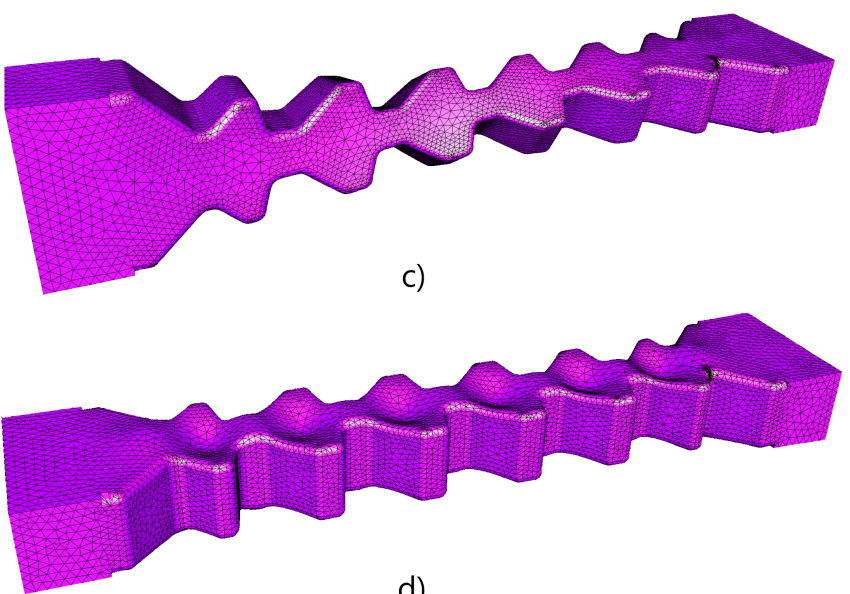

d)

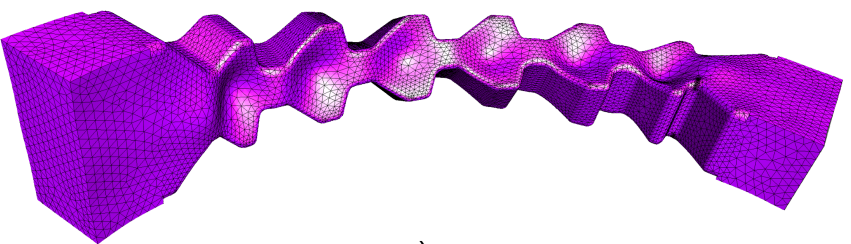

e)

Fig. 2. Example of more complex shape deformations: a) original structure, b) bending, c) twisting $90^{\circ}$, d) squeezing resonant cavities vertically, e) squeezing + twisting + bending

the deforming operation (position and displacements of the control points). Shape deformation is performed on the volume mesh generated for the primal structure. The deformed structure is then evaluated by an EM simulator to calculate its response in the frequency domain (S-parameters) or the frequencies and Q-factors of the resonating modes with an eigenmode solver. Optimization of the structure continues until termination conditions are met (e.g., error value below a preset threshold or maximum number of iterations reached). The final mesh of the deformed structure can be converted to a 3D model in STL file format and further processed in 3D geometry modeling software-for example, to prepare production files for additive manufacturing. 


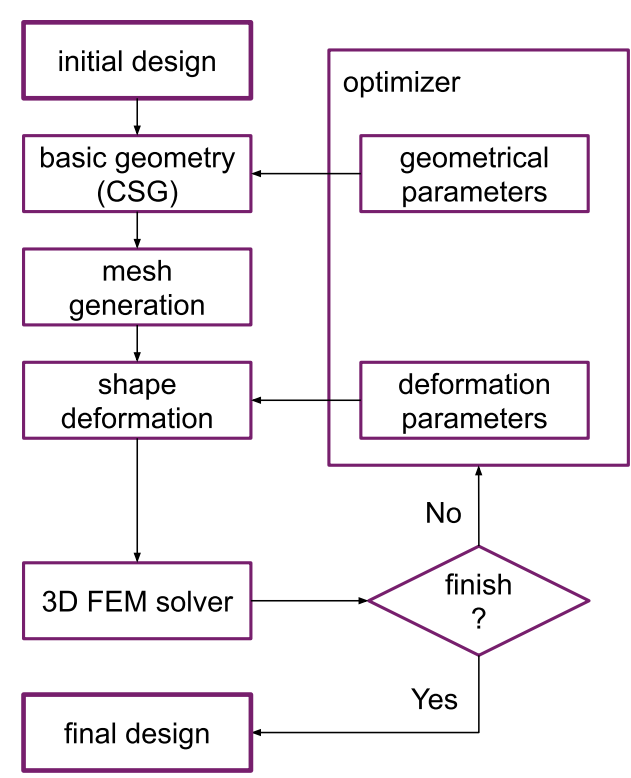

Fig. 3. A flowchart representing the general design optimization workflow.

\section{Optimization of CAVity Resonators Using Shape DEFORMATIONS}

The deformation scheme was used to optimize cavity resonators with enhanced electromagnetic performance. The goal was to investigate whether shape deformation can improve the selected electrical parameters. In particular, we focused on maximizing the quality factor and higher-order mode separation. Let us consider the simple example of a rectangular cavity resonator with rounded edges presented in Fig. 4 . In order to alter the geometry of the structure, a set of control points is selected. In this example, a $3 \mathrm{D}$ grid of points is again defined, consisting of a total of 26 control points, as shown in Fig. $4 \mathrm{p}$. The non-zero displacement vectors are then defined for some of these points. Here, two offset vectors are specified at

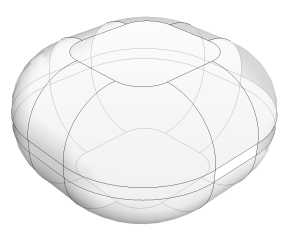

a)
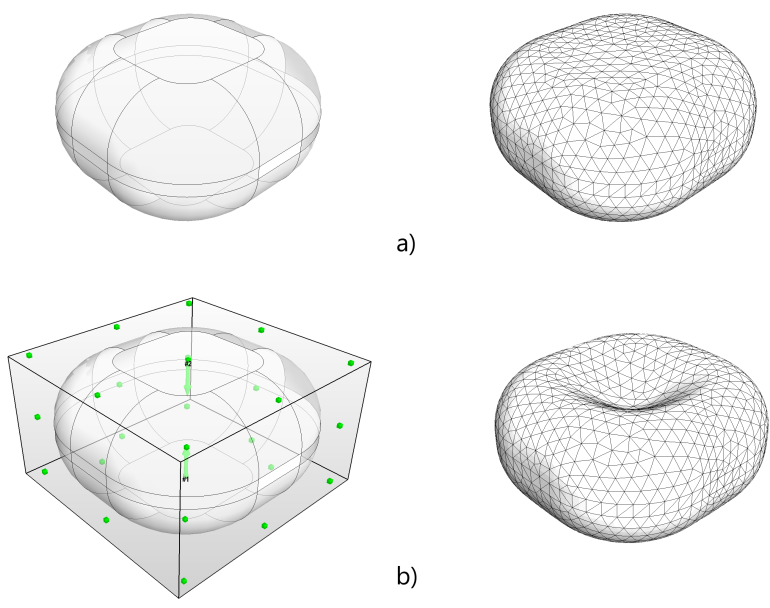

b)

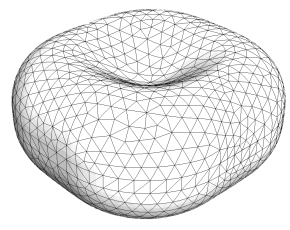

Fig. 4. Example of shape deformation: a) basic rectangular cavity with rounded edges and its mesh, b) definition of a squeezing operation and resulting mesh. Green arrows show the direction of the deformation, and green dots stand for fixed points. the center of the cavity's top and bottom planes, pointing into the cavity. Given the list of control points and displacement vectors, the deformation field can be evaluated and applied to the volume mesh, resulting in a smoothly deformed geometry, also shown in Fig. 4p. The deformed mesh is then used by a 3D FEM electromagnetic field solver [21] to perform eigenmode analysis in order to calculate the frequencies and quality factors of the modes excited in the structure.

\section{A. Investigation of $Q$ vs. Spurious-Free Performance}

In this experiment, the goal was to obtain the highest possible separation $(\Delta f)$ of the first higher-order mode resonating at $f_{1}$ from the fundamental one at $f_{0}$, while also achieving a given quality factor $Q$ and preserving the resonant frequency at $f_{0}$. In the simulations, we assumed finite conductivity of the cavity corresponding to that of aluminum $\left(\sigma=3.8 \cdot 10^{7}\right.$ $\mathrm{S} / \mathrm{m}$ ) and a resonant frequency equal to $f_{0}=10 \mathrm{GHz}$. The optimization loop involves managing two sets of design variables: geometrical parameters altering the basic shape of the primal structure (cavity height, length, and width), and deformation parameters controlling the deforming operation; in this study, only deformation along the Z-axis (squeezing in the vertical direction) was considered. A quasi-Newton gradient optimization was used in this resonant cavity design workflow.

The optimization was run several times for different values of $Q$ and $\Delta f$, which resulted in several resonator geometries, all having the same resonant frequency $f_{0}=10 \mathrm{GHz}$ and various Q-factors and spurious mode separations. The results are summarized in Fig. 5 in the form of a trade-off chart, showing an achievable $Q$ vs. mode separation $\Delta f=f_{1}-f_{0}$. It can be observed that a greater higher-order mode separation can be achieved at the cost of lowering the Q-factor. Depending on the particular application, a resonator shape realizing the desired $Q$ and $\Delta f$ can be selected.

\section{B. Cavity Resonators with Extended Spurious-Free Region}

A second test was carried out to further demonstrate the possibilities of resonant cavity design based on user-defined

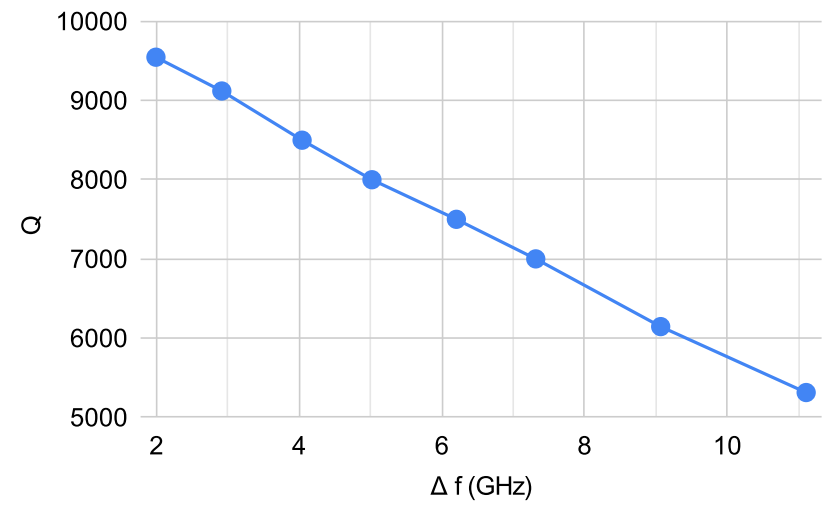

Fig. 5. Trade-off chart: resonator Q-factor vs. higher-order mode separation for $\sigma=3.8 \cdot 10^{7} \mathrm{~S} / \mathrm{m}$. 
TABLE I

EM-SIMULATED SPECIFICATIONS OF THE CAVITY RESONATORS

\begin{tabular}{|l|c|c|c|c|}
\hline Project & $f_{0}(\mathrm{GHz})$ & $f_{1}(\mathrm{GHz})$ & $f_{1} / f_{0}$ & $Q_{u}$ \\
\hline \hline Hemispherical & 10 & 19.8 & 1.98 & 7100 \\
\hline This work (shape deform.) & 10 & 20.2 & 2.02 & 7100 \\
\hline
\end{tabular}

shape deformation. For this reason, a recently reported highQ cavity resonator with an extended spurious-free region was examined [26]. This design was a geometrically shaped hemispherical cavity prepared using the CSG approach by performing a number of Boolean operations and edge filleting. For comparison, a new deformed cavity resonator was designed using the shape deformation approach to outperform the simulation results achieved in [26]. An optimization procedure was run using the framework described in Section III] to obtain the greatest possible mode separation while maintaining the Qfactor. The basic rectangular cavity with optimized dimensions of $18.77 \times 18.77 \times 12.72 \mathrm{~mm}$ and filleted edges was squeezed vertically from top and bottom. The two central control points were moved towards the center of the cavity by $5 \mathrm{~mm}$ each. The final shape is the result of a RBF interpolation with a Gaussian spline of radius $r=4 \mathrm{~mm}$, as in (3). A 3D model of the resulting structure is provided in Fig. 6. The comparison of $Q$ and $f_{1} / f_{0}$ for both simulated models is summarized in Table [I The unloaded quality factor $Q_{u}$ achieved by both designs was 7100 for metal conductivity $\sigma=5.97 \cdot 10^{7} \mathrm{~S} / \mathrm{m}$ (copper). The resonant frequency of the hemispherical cavity was 10 $\mathrm{GHz}$, and the spurious-free region went up to $19.8 \mathrm{GHz}$. In this study, geometric deformation gave a greater spurious mode separation (up to $20.2 \mathrm{GHz}$ ), while preserving the Q-factor. The electrical performance achieved is slightly better than in the compared work, but a very basic model deformation was performed, rather than several geometrical operations.

\section{Design of Waveguide Cavity Filters using Shape DEFORMATIONS}

These two cavity-resonator examples have shown that shape deformation can be successfully applied to the CAD of simple

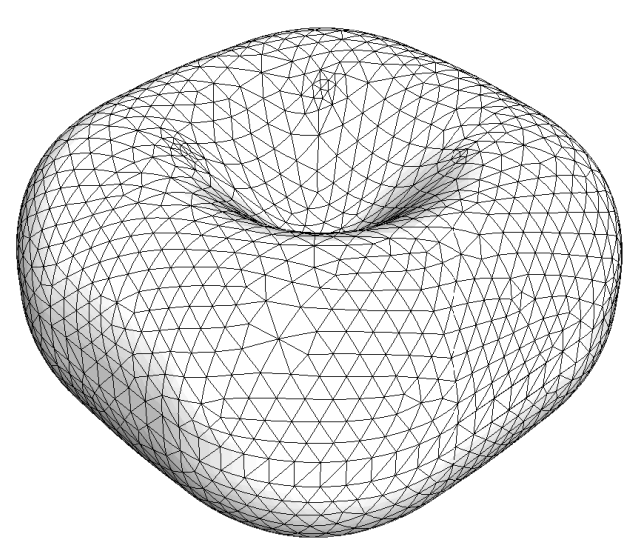

Fig. 6. Shape of the cavity resonator with $f_{0}=10 \mathrm{GHz}, \Delta f=10.2 \mathrm{GHz}$ and $Q=7100$ (copper, $\sigma=5.97 \cdot 10^{7} \mathrm{~S} / \mathrm{m}$ ). microwave resonators, yielding enhanced electromagnetic performance. This approach should thus be used to design more complex components, such as waveguide cavity filters, in order to demonstrate its full potential in practical applications. We will consider two filter designs of Ku-band broadband input filters for satellite communication systems [27]. In these new designs, we want to achieve an improvement in terms of outof-band rejection and spurious-free performance by applying RBF-based shape deformation to the basic filter geometry. The prototypes are to be additively manufactured as single pieces, and this fabrication technology imposes certain constraints on the allowable shape. This has to be taken into account very early on, even before the shape is deformed. It has to be noted that the Q-factor of the fabricated filter cavities will be limited due to the surface roughness and the conductivity that can be achieved in AM technology. In our designs, the $Q$ and the actual metal conductivity obtained in the AM process will be estimated by investigation of the insertion loss of the fabricated structures. Note that, although microwave filters with better spurious-free performance have already been reported for other technologies (such as combline, ceramic, and coplanar waveguide filters [1], [28]-[32]), this work focuses on presenting the improvements achieved with a simple geometry deformation, allowing the final structure to be 3D-printed in one piece without internal supports.

\section{A. Third-Order Bandpass Filter with a Complex Shape}

The first structure shown is a third-order cavity bandpass filter (BPF) with inline topology. The reference structure and the starting point for our design is the conventional third-order BPF shown in Fig. 77. The main specifications to be met are

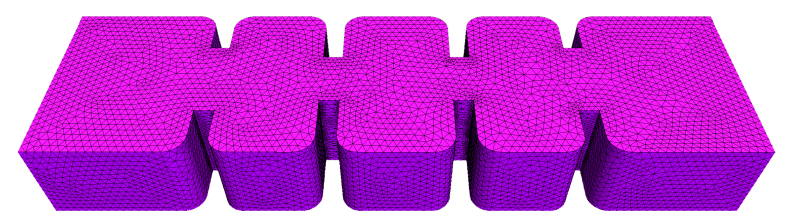

a)

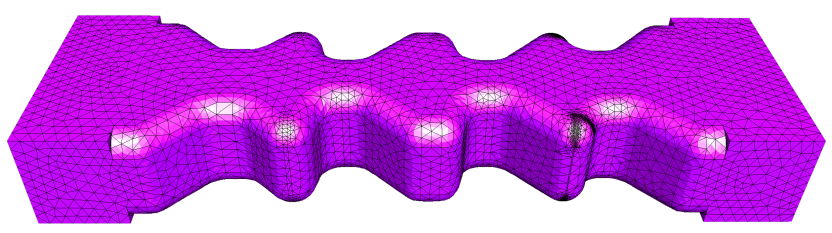

b)

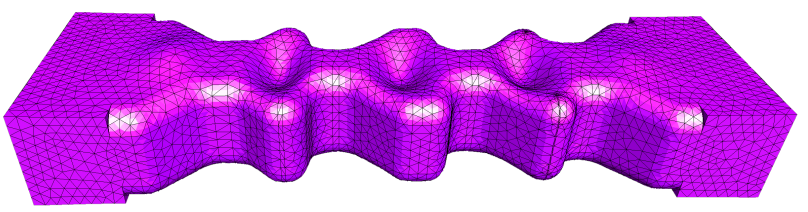

c)

Fig. 7. Geometry of a third-order bandpass filter: a) standard structure, b) structure prepared for AM manufacturing, c) final structure with cavity deformation. 
a center frequency of $f_{0}=14.25 \mathrm{GHz}$, bandwidth equal to $500 \mathrm{MHz}$ (fractional bandwidth $\mathrm{FBW}=3.5 \%$ ), and return loss (RL) in the passband over $20 \mathrm{~dB}$. Even though shape deformation techniques allow shapes that are optimal in terms of performance to be obtained, several practical constraints have to be taken into account during the design process. As the goal is to enable $3 \mathrm{D}$ printing as a single manifold, the filter model must accommodate the constraints of the SLM process. Should the structure be tilted at an angle greater than $45^{\circ}$ along the printing axis, extra supporting posts need to be placed inside the model. These supports then need to be removed later in a post-printing step, which could be a troublesome operation. A standard waveguide filter, shown in Fig. 7a, is not compliant with the SLM process. For this reason, the primal filter model designed in this way is modified to meet the requirements of AM and to avoid the need to insert supporting posts inside the structure during printing; this is why the resonators have a quasi-triangular shape (see Fig. $7 \mathrm{p}$ ).

Shape deformation was then used to squeeze the top and bottom of the resonator surfaces. In this case, the deformation depth of the cavities is $2.5 \mathrm{~mm}$. As with the previous resonant cavities, shape deformation involves Gaussian spline RBFs (3). At this stage, the design procedure involved geometric optimization, which included the design parameters of the basic structure (length of the resonators, widths of the irises) and simultaneous shape deformation. The zero-pole method was used as the optimization technique [33], [34]. The entire process was performed in the InventSim EM simulator within the newly developed module allowing efficient design optimization with simultaneous shape deformation, including the optimization of control point positions and their movements. The final shape of the designed filter is shown in Fig. 77.

The simulation results for the final third-order filter are presented in Fig. 8. The response of the standard H-plane filter design is also plotted for comparison. It can be observed that the cavity deformation resulted in an approximately $1.5 \mathrm{GHz}$ wider spurious-free region than the reference design. Our experiments show that the deeper the squeeze of the cavity,

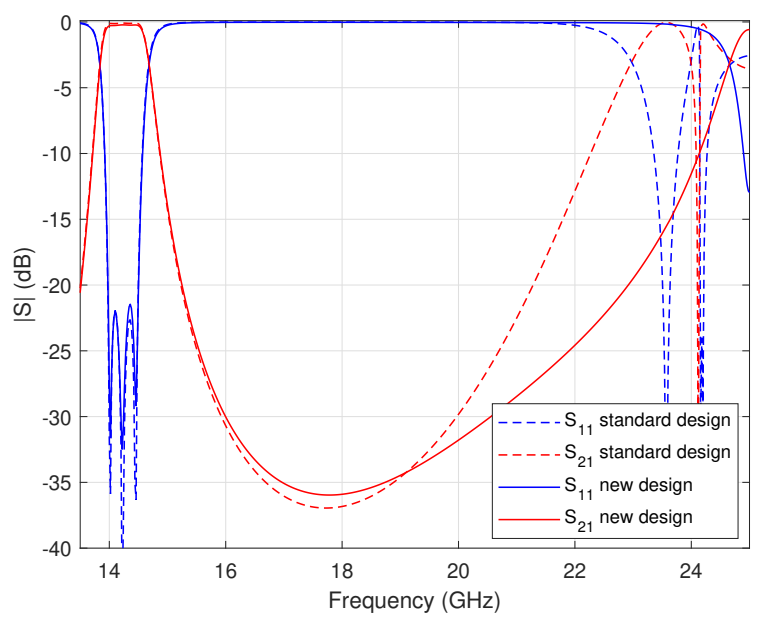

Fig. 8. Simulation comparison of the third-order BPF: standard design vs. deformed design.

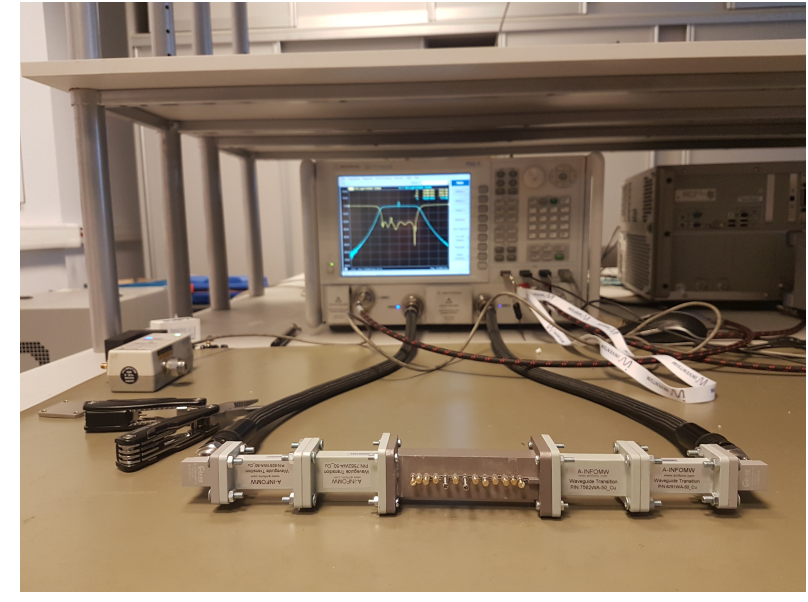

Fig. 9. Measurement setup using WR-51-to-WR-75 waveguide adapters. The sixth-order filter is measured here.

the greater the spurious mode separation.

A prototype of the new filter design was manufactured as a single mechanical part using the SLM process [2], [35] with a TruPrint 1000 printer from Trumpf with an aluminumsilicon-based alloy AlSi10Mg, and then measured. In order to observe the wideband performance of the filter, waveguide adapters WR-51-to-WR-75 were used to reduce the influence of higher order modes excited in the WR-75 waveguide-tocoax adapters. The measurement setup using a vector network analyzer (VNA) and adapters is shown in Fig. 9. The simulations and measurements of the printed prototype are compared in Fig. 10. The measurements are in good agreement with the simulations. The insertion loss (IL) at center frequency $f_{0}$ is $0.2 \mathrm{~dB}$, the RL is greater than $20 \mathrm{~dB}$ and the spuriousfree region reaches up to $24.5 \mathrm{GHz}$. The response of the filter with no tuning screws is also provided. It should be noted that the initial measured response (without tuning) is

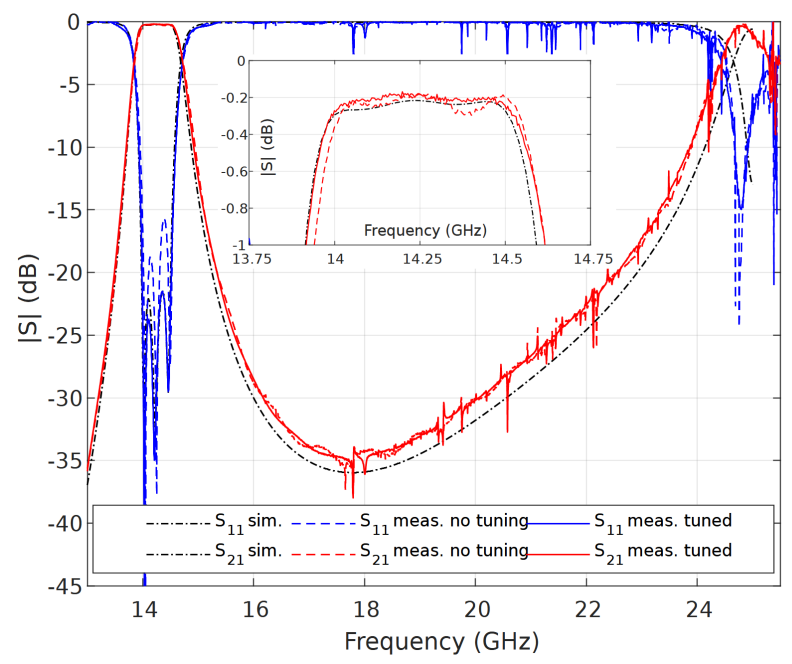

Fig. 10. Wideband comparison of simulations and measurement of the thirdorder deformed cavity BPF: simulation results: black dash-dotted lines; measurements with no tuning screws: dashed blue-red lines; final measurements (tuned): solid blue-red lines. 
very satisfactory: the IL at $f_{0}$ and spurious-free region match the simulations, and the RL in the passband is over $15 \mathrm{~dB}$. Tuning screws were used to improve the in-band RL to the desired level of $20 \mathrm{~dB}$. Based on the IL of the printed filter, we have estimated the effective conductivity of the 3D-printed aluminum powder, which according to these measurements is about $\sigma_{\text {eff }}=4.2 \cdot 10^{6} \mathrm{~S} / \mathrm{m}$. This value is comparable to other reported aluminum structures fabricated with SLM-for example. it is slightly better than the effective conductivity achieved in [36]. This estimated conductivity $\sigma_{\text {eff }}$ was later used in EM simulations of subsequent designs. The measurements were also used to determine the approximate Q-factor of the fabricated filter, which is equal to $Q=1800$.

\section{B. Sixth-Order Bandpass Filter with a Complex Shape}

Upon completion of the third-order filter prototype, a sixthorder cavity BPF design was chosen for preparation. The specifications for the new design match those of the third-order filter: a center frequency of $f_{0}=14.25 \mathrm{GHz}, \mathrm{FBW}=3.5 \%$ and $\mathrm{RL}>20 \mathrm{~dB}$ in the passband. Again, the basic filter geometry, shown in Fig. 11, is modified to comply with the 3D printing process, resulting in the triangle-shaped model presented in Fig. $11 \mathrm{p}$. To achieve greater spurious mode separation in the stopband, the deformation of the cavities is deeper and equals $3.75 \mathrm{~mm}$. EM simulations of the sixth-order BPF are computed for the estimated metal conductivity $\sigma_{e f f}=4.2 \cdot 10^{6} \mathrm{~S} / \mathrm{m}$, equivalent to what was previously obtained with the third-order prototype. A comparison of the simulated wideband responses of the reference design and the new model is provided in Fig. 12. Here, the spurious-free region is up to ca. $27.8 \mathrm{GHz}$, and so equal to $1.95 f_{0}$ and approximately $4.5 \mathrm{GHz}$ wider than in the traditional cavity BPF design. Note that the new filter model gives very high insertion loss in the stopband, which is another advantage of the deformation-based design. A prototype of the filter from Fig. 11p was fabricated using SLM technology the same way as the previous model and measured using the same setup shown in Fig. 9. The measurement results are plotted in Fig. 13a (passband response) and Fig. 13b (wideband response). The passband comparison allows us to observe that the filter response is satisfactory and consistent with the simulations. The measurement taken in a wider band becomes unclear above about $26 \mathrm{GHz}$, due to the limited

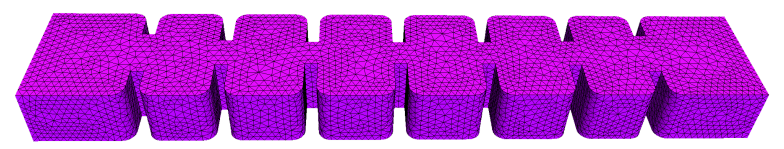

a)

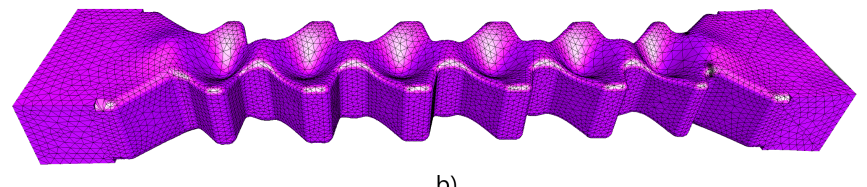

b)

Fig. 11. Geometry of a sixth-order bandpass filter: a) standard structure, b) deformed structure.

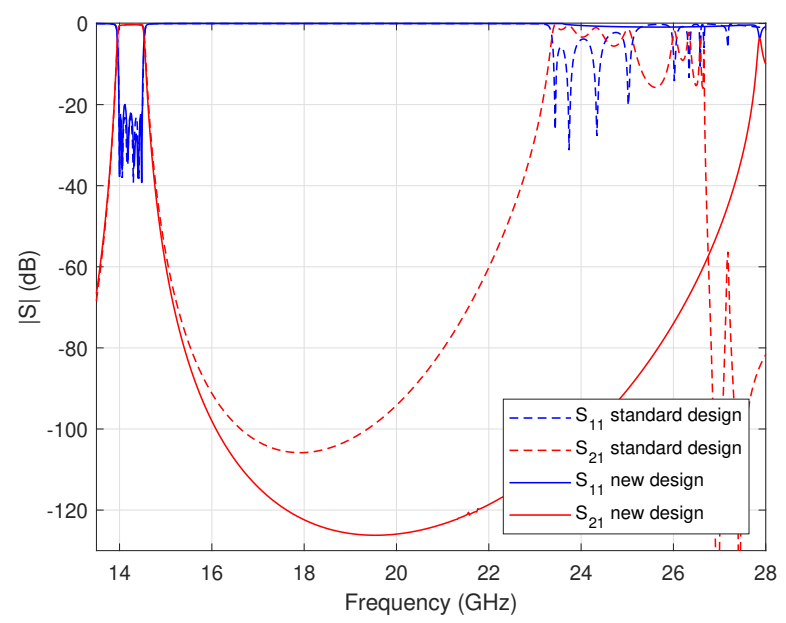

Fig. 12. Simulation comparison of the sixth-order BPF: standard design vs. deformed design.

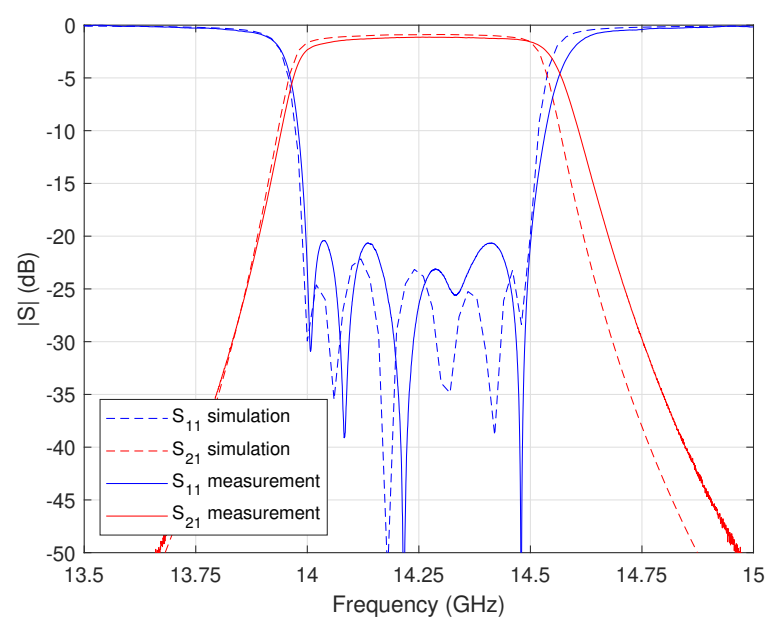

(a)

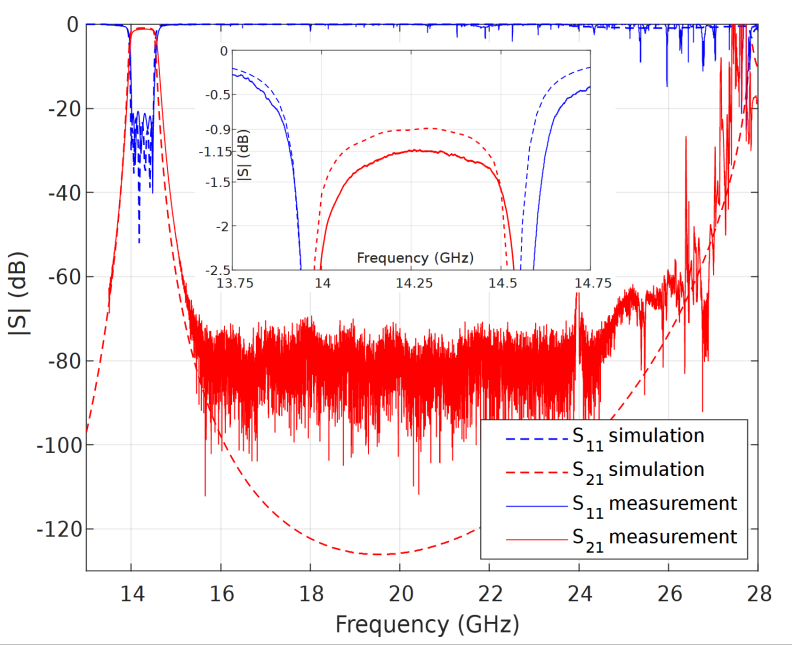

(b)

Fig. 13. Comparison of simulations and measurement for the sixth-order deformed cavity BPF. (a) passband response. (b) wideband response. 
operating band of the waveguide adapters used. Nevertheless, from this measurement it can be discerned that the out-of-band characteristics match the response provided by EM simulation. The measured IL in the passband is $1.15 \mathrm{~dB}$, which is slightly higher than the expected $0.9 \mathrm{~dB}$ received from the simulation with effective metal conductivity $\sigma_{e f f}$. This is mostly caused by the addition of tuning screws which were not accounted for in the simulations. The estimated Q-factor of the filter equals $Q=930$. One way of reducing the losses and receiving a higher Q-factor could be the metalization of all surfaces of the filter in a post-printing step. This could be either silver, copper, or gold plating, depending on the fabrication process. 3D-printed, copper, and gold plated waveguide components with an effective conductivity of copper $\sigma_{C u}=2.04 \cdot 10^{7} \mathrm{~S} / \mathrm{m}$ and gold $\sigma_{A u}=1.24 \cdot 10^{7} \mathrm{~S} / \mathrm{m}$ are reported in [37]. For such conductor losses, the estimated Q-factor of the presented sixthorder BPF would be equal to $Q_{C u}=2550$ or $Q_{A u}=1970$, respectively. Silver plating is presumed to bring similar, if not better, improvements, and should be considered in further research.

\section{CONCLUSION}

This paper introduced and experimentally verified a shape deformation technique for the CAD of microwave components. This approach opens up new modeling possibilities to RF $\&$ microwave engineers by offering more degrees of freedom than the common CSG approach. The proposed deformation of 3D solids is easy to couple with known EM CAD tools and is intuitive thanks to the idea of altering the shape by moving arbitrarily chosen control points. First, the novel cavity resonators and filters with enhanced electromagnetic performance were designed using this deformation strategy and manufactured in a single mechanical part with $3 \mathrm{D}$ printing. These prototypes were used to verify the designs and to test the quality of AM with SLM technology for this category of high-frequency components. The measurement results confirmed the effectiveness of the new deformation-based design approach and demonstrated the possibilities offered by $3 \mathrm{D}$ printing. The deficiencies of this fabrication process, such as deteriorated metal conductivity, were also recognized. Future research directions can include the further investigation of complex microwave structures (filters, couplers, antennas) employing shape deformation, as well as enhancing the AM process by silver-plating the printed components.

\section{REFERENCES}

[1] P. Booth and E. V. Lluch, "Enhancing the performance of waveguide filters using additive manufacturing," Proc. IEEE, vol. 105, no. 4, pp. 613-619, April 2017.

[2] F. Calignano, D. Manfredi, E. P. Ambrosio, S. Biamino, M. Lombardi, E. Atzeni, A. Salmi, P. Minetola, L. Iuliano, and P. Fino, "Overview on additive manufacturing technologies," Proc. IEEE, vol. 105, no. 4, pp. 593-612, April 2017.

[3] T.-H. Chio, G.-L. Huang, and S.-G. Zhou, "Application of direct metal laser sintering to waveguide-based passive microwave components, antennas, and antenna arrays," Proc. IEEE, vol. 105, no. 4, pp. 632 644, April 2017.

[4] C. Tomassoni, O. A. Peverini, G. Venanzoni, G. Addamo, F. Paonessa, and G. Virone, "3D printing of microwave and millimeter-wave filters: Additive manufacturing technologies applied in the development of highperformance filters with novel topologies," IEEE Microw. Mag., vol. 21, no. 6 , pp. 24-45, June 2020 .
[5] S. Ghosh and S. Lim, "Perforated lightweight broadband metamaterial absorber based on 3-D printed honeycomb," IEEE Antennas Wireless Propag. Lett., vol. 17, no. 12, pp. 2379-2383, Dec. 2018.

[6] J. J. P. Venter, R. Maharaj, and T. Stander, "Additive manufacturing of interdigital filters with arbitrary line cross section," IEEE Trans. Compon. Packag. Manuf. Technol., vol. 10, no. 4, pp. 686-693, April 2020.

[7] K. Lomakin, L. Klein, L. Ringel, J. Ringel, M. Sippel, K. Helmreich, and G. Gold, "3D printed E-band hybrid coupler," IEEE Microw. Wireless Compon. Lett., vol. 29, no. 9, pp. 580-582, Sept. 2019.

[8] H. García-Martínez, E. Avila-Navarro, G. Torregrosa-Penalva, N. Delmonte, L. Silvestri, S. Marconi, G. Alaimo, F. Auricchio, and M. Bozzi, "Design and fabrication of a band-pass filter with EBG single-ridge waveguide using additive manufacturing techniques," IEEE Trans. Microw. Theory Techn., vol. 68, no. 10, pp. 4361-4368, Oct. 2020.

[9] E. López-Oliver and C. Tomassoni, "3-D-printed dual-mode filter using an ellipsoidal cavity with asymmetric responses," IEEE Microw. Wireless Compon. Lett., vol. 31, no. 6, pp. 670-673, June 2021.

[10] T. W. Sederberg and S. R. Parry, "Free-form deformation of solid geometric models," SIGGRAPH Comput. Graph., vol. 20, no. 4, p. 151-160, Aug. 1986.

[11] J. Gain and D. Bechmann, "A survey of spatial deformation from a usercentered perspective," ACM Trans. Graph., vol. 27, no. 4, Oct. 2008.

[12] R. Noble and G. Clapworthy, "Direct manipulation of surfaces using nurbs-based free-form deformations," in 1999 IEEE Int. Conf. Inf. Vis. (IV), 1999, pp. 238-243.

[13] D. Forti and G. Rozza, "Efficient geometrical parametrisation techniques of interfaces for reduced-order modelling: application to fluid-structure interaction coupling problems," Int. J. Comput. Fluid Dyn., vol. 28, no. 3-4, SI, pp. 158-169, 2014

[14] A. Koshakji, A. Quarteroni, and G. Rozza, "Free form deformation techniques applied to 3D shape optimization problems," Commun. Appl. Ind. Math., vol. 4, 2013.

[15] B. Zhang, Z. Feng, B. Xu, and T. Yang, "Efficient aerodynamic shape optimization of the hypersonic lifting body based on free form deformation technique," IEEE Access, vol. 7, pp. 147 991-148 003, 2019.

[16] T. Henneron, A. Pierquin, and S. Clénet, "Mesh deformation based on radial basis function interpolation applied to low-frequency electromagnetic problem," IEEE Trans. Magn., vol. 55, no. 6, pp. 1-4, June 2019.

[17] N. Mahdi, S. Bila, S. Verdeyme, M. Aubourg, K. Khoder, A. Bessaudou, F. Cosset, C. Durousseau, J. Puech, L. Estagerie, D. Pacaud, and H. Leblond, "A shape optimization library for the design of microwave components," Int. J. Microw. Wirel. Technol., vol. 6, pp. 31-37, Feb. 2014.

[18] H. Khalil, S. Bila, M. Aubourg, D. Baillargeat, S. Verdeyme, F. Jouve, and T. Chartier, "Shape optimization of a dielectric resonator for improving its unloaded quality factor," Int. J. RF and Microw. CAE, vol. 21, no. 1, pp. 120-126, Jan. 2011.

[19] A. Dia, C. Durousseau, C. Menudier, L. Carpentier, O. Ruatta, and S. Bila, "Shape optimization of a compact dual-mode filter using Bézier curves parametrization," in 2021 IEEE MTT-S Int. Microw. Filter Workshop (IMFW), 2021, pp. 137-139.

[20] J. Jin, F. Feng, J. Zhang, J. Ma, and Q.-J. Zhang, "Efficient EM topology optimization incorporating advanced matrix padé via lanczos and genetic algorithm for microwave design," IEEE Trans. Microw. Theory Techn., vol. 69, no. 8, pp. 3645-3666, Aug. 2021.

[21] "Inventsim 3D FEM electromagnetic field solver, ver. 3.0," 2021.

[22] M. Baranowski, L. Balewski, A. Lamecki, and M. Mrozowski, "Design of cavity resonators applying shape deformation techniques," in 2021 IEEE MTT-S Int. Microw. Filter Workshop (IMFW), 2021, pp. 291-293.

[23] Z. Levi and D. Levin, "Shape deformation via interior RBF," IEEE Trans. Vis. Comput. Graphics, vol. 20, no. 7, pp. 1062-1075, July 2014.

[24] A. de Boer, M. van der Schoot, and H. Bijl, "Mesh deformation based on radial basis function interpolation," Comput. Struct., vol. 85, no. 11, pp. 784-795, June 2007.

[25] M. Botsch and L. Kobbelt, "Real-time shape editing using radial basis functions," Comput. Graph. Forum, vol. 24, no. 3, pp. 611-621, Sept. 2005.

[26] J. Li and T. Yuan, "A geometrically shaped hemispherical cavity resonator with extended spurious-free region," in 2020 IEEE MTT-S Int. Microw. Symp., 2020, pp. 731-734.

[27] P. Vallerotonda, L. Pelliccia, C. Tomassoni, F. Cacciamani, R. Sorrentino, J. Galdeano, and C. Ernst, "Compact waveguide bandpass filters for broadband space applications in C and Ku-bands," in 2019 Eur. Microw. Conf. Cent. Eur. (EuMCE), 2019, pp. 116-119. 
[28] H.-W. Yao, K. Zaki, A. Atia, and T. Dolan, "Improvement of spurious performance of combline filters," in 1997 IEEE MTT-S Int. Microw. Symp. Dig., vol. 2, 1997, pp. 1099-1102 vol.2.

[29] F. Gentili, L. Pelliccia, R. Sorrentino, and G. Bianchi, "High Q-factor compact filters with wide-band spurious rejection," in 2012 42nd Eur. Microw. Conf. (EuMC), 2012, pp. 160-163.

[30] E. López-Oliver, C. Tomassoni, L. Silvestri, M. Bozzi, L. Perregrini, S. Marconi, G. Alaimo, and F. Auricchio, "3-D-printed compact bandpass filters based on conical posts," IEEE Trans. Microw. Theory Techn., vol. 69, no. 1, pp. 616-628, Jan. 2021.

[31] I. Hunter, S. Afridi, and M. Sandhu, "Integrated ceramic waveguide filters with improved spurious performance," in 2015 Eur. Microw. Conf. (EuMC), 2015, pp. 674-677.

[32] S.-G. Mao and Y.-Z. Chueh, "Coplanar waveguide bandpass filters with compact size and wide spurious-free stopband using electromagnetic bandgap resonators," IEEE Microw. Wireless Compon. Lett., vol. 17 no. 3, pp. 181-183, March 2007.

[33] P. Kozakowski and M. Mrozowski, "Automated CAD of coupled resonator filters," IEEE Microw. Wireless Compon. Lett., vol. 12, no. 12, pp. 470-472, Dec. 2002.

[34] L. Balewski, G. Fotyga, M. Mrozowski, M. Mul, P. Sypek, D. Szypulski, and A. Lamecki, "Step on it!: Bringing fullwave finite-element microwave filter design up to speed," IEEE Microw. Mag., vol. 21, no. 3 , pp. 34-49, Mar. 2020.

[35] O. A. Peverini, M. Lumia, F. Calignano, G. Addamo, M. Lorusso, E. P. Ambrosio, D. Manfredi, and G. Virone, "Selective laser melting manufacturing of microwave waveguide devices," Proc. IEEE, vol. 105, no. 4, pp. 620-631, April 2017.

[36] F. Zhang, C. Guo, Y. Zhang, Y. Gao, B. Liu, M. Shu, Y. Wang, Y. Dong, M. J. Lancaster, and J. Xu, "A 3-D printed bandpass filter using $\mathrm{TM}_{211}$ mode slotted spherical resonators with enhanced spurious suppression," IEEE Access, vol. 8, pp. 213 215-213 223, 2020.

[37] A. I. Dimitriadis, T. Debogović, M. Favre, M. Billod, L. Barloggio, J.P. Ansermet, and E. de Rijk, "Polymer-based additive manufacturing of high-performance waveguide and antenna components," Proc. IEEE, vol. 105, no. 4, pp. 668-676, April 2017. 